\title{
Raising awareness in university students of languages
}

\section{[La conscientisation des apprenants de langue au niveau universitaire]}

\author{
Meta Lah
}

\section{DOI: 10.18355/XL.2017.10.04.26}

\begin{abstract}
Résumé
Dans le présent article, nous nous proposons d'abord d'examiner les notions d'autonomie et de conscientisation de l'apprenant en classe de langue ; un apprenant autonome étant celui qui est capable de se charger de son propre apprentissage et d'avoir une attitude critique envers le processus pédagogique et le contenu des cours et la conscientisation, selon Plathner, un processus, travail sur la conscience de l'apprenant. Dans la deuxième partie, nous décrivons l'expérience que nous avons menée avec les étudiants : la rédaction des comptes-rendus après chaque cours. À la fin des cours, nous avons demandé aux étudiants d'écrire une réflexion pour évaluer cette activité. Nous avons reçu 50 réflexions, 23 d'étudiants au niveau licence et 27 des étudiants au niveau Master (futurs enseignants en formation initiale). L'hypothèse de départ était qu'il y aurait dans les réflexions des étudiants de Master plus de commentaires concernant le processus d'enseignement/apprentissage, ce qui montrerait qu'ils sont plus conscients de ce processus - ce qui s'est finalement avéré. À la fin, nous proposons plusieurs changements, pour chacun des deux groupes séparément.
\end{abstract}

Mots-clés : apprenants de FLE au niveau universitaire, autonomie, conscientisation, auto-observation, comptes-rendus

\section{Introduction}

Depuis l'apparition de l'approche communicative, le centre de l'intérêt dans l'enseignement des langues étrangères est mis sur l'apprenant. De nombreux auteurs soulignent l'importance de ce changement dans l'enseignement/apprentissage, pas seulement des langues mais de toutes les disciplines. Selon Cuq et Gruca (2002 : 247), il s'agit d' « un changement radical en ce qui concerne la conception de l'apprentissage (...) qui met la participation de l'apprenant au coeur de l'apprentissage, ce dernier étant considéré comme un processus actif et créateur dont le rythme dépend de l'individu ». Udier et al. (2016 : 103) soulignent que le fait de mettre l'apprenant au centre du processus a aujourd'hui une valeur axiomatique et que l'enseignement n'est plus censé être une transmission du savoir de l'enseignant à l'apprenant mais plutôt une production active du savoir. L'apprenant est considéré comme un participant actif dans le processus. De son côté, Hanesova (2015 : 46) ajoute que, même si cela peut paraître cliché, l'enseignement/apprentissage, centré sur l'apprenant est sans aucun doute plus efficace que l'enseignement/apprentissage, centré sur l'enseignant.

Dans le présent article, nous nous proposons d'examiner tout d'abord les notions d'autonomie et de conscientisation de l'apprenant. Les deux notions sont fortement entreliées; en classe de langue, on essaie de conscientiser l'apprenant pour le rendre plus autonome dans son apprentissage et lui permettre de mieux travailler et le rendre plus proche du processus et du contenu de l'enseignement/apprentissage (Vieira et al., 2008 : 219). Dans la deuxième partie de l'article, nous décrirons une expérience concrète qui, d'après nous, pourrait mener à la conscientisation des apprenants et à un travail plus autonome en classe de langue. Nous allons nous focaliser - mais pas uniquement - sur une expérience pratique à l'aide de laquelle nous avons voulu voir à quel point les apprenants au niveau universitaire peuvent suivre les cours et repérer les 
objectifs de l'enseignant. L'activité que nous avons utilisée pour les accompagner le long de l'année est la rédaction de comptes-rendus. Dans l'analyse, nous décrirons les avantages et les points à améliorer, cités par les étudiants. Nous allons aussi essayer de donner des suggestions pour utiliser ces comptes-rendus de manière plus efficace, comme un outil de conscientisation et peut-être même comme un outil d'encadrement de l'apprentissage, comme le font Temperman et al. (2007).

\section{L'autonomie en classe de langue}

Dans son article Autonomy in language teaching and learning, Benson fait passer en revue la bibliographie concernant l'autonomie dans l'enseignement/apprentissage. Il constate qu'au début du 21e siècle, l'intérêt pour le sujet a beaucoup augmenté. Le nombre d'unités, publiées entre 2000 et 2006 dépasse le nombre des publications des 25 années précédentes (Benson, $2006: 21$ ). Avec la parution du Cadre européen de référence pour les langues en 2001, le concept de l'autonomie devient encore plus actuel.

Qu'est-ce que l'autonomie ? Holec (1979: 3), un des premiers auteurs à parler de ce concept, reprend les définitions du dictionnaire et constate que l'autonomie est une capacité, " puissance de faire quelque chose » et non une conduite, "façon d'agir ". Selon lui, l'autonomie est « une compétence potentielle de comportement dans une situation donnée, celle de l'apprentissage, en l'occurence " (ibid.) Il ajoute qu'un apprenant est autonome quand il est capable de prendre en charge son apprentissage et que cet apprentissage est donc un apprentissage autodirigé ou en autonomie (ibid. : 4). Little (1991 : 4) explique que " essentiellement, l'autonomie est la capacité de se détacher, de réfléchir de façon critique, de prendre des décisions et d'agir indépendamment ». Selon lui, l'apprenant doit aussi développer une sorte de relation psychologique envers le processus et le contenu de ce qu'il apprend (ibid.) Nous pourrions parler de plusieurs niveaux d'autonomie de l'apprenant - Nunan (1997), en établit cinq : le niveau de conscience, de participation, d'intervention, de création et de transcendence - ainsi que de plusieurs caractéristiques de l'apprenant autonome selon Dickinson (1993 : 330), il y en a cinq : l'apprenant autonome est conscient de ce que fait l'enseignant et capable de reconnaître les objectifs de l'enseignement, il est capable de formuler/fixer ses propres objectifs, il est capable de choisir des stratégies d'apprentissage appropriées, souvent de façon consciente, il peut surveiller l'utilisation de ses stratégies d'apprentissage et il peut identifier les stratégies qui ne fonctionnent pas pour lui. Wenden (1991: 53) ajoute qu'un apprenant autonome devrait se poser la question sur ce qu'apprendre une langue étrangère signifie pour lui et cite deux de ses attitudes : la volonté de prendre la responsabilité de son apprentissage et la confiance en sa capacité d'apprenant, cela veut dire que les apprenants croient en leur capacité d'apprendre et de gérer leur apprentissage. Cotterall (2008 : 1-2) reprend les définitions les plus répandues, celles de Holec, Little et Benson, et constate qu'on pourrait parler de trois niveaux d'autonomie : le niveau méthodologique (Holec) l'apprenant est capable de prendre en charge son propre apprentissage, le niveau psychologique (Little) et le niveau contenu (Benson) : le contenu de l'apprentissage devrait librement être déterminé par les apprenants.

L'autonomie est-elle un concept universel ou est-elle culturellement marquée ? Elle a longtemps été influencée par l'idéologie des locuteurs natifs des pays sources et considérée comme un concept de l'Ouest (Adamson et Sert, 2012 : 23). De nombreux auteurs mentionnent les apprenants des autres cultures comme passifs. Chan (1999, cité dans Comerio, 2014 : 454), par exemple, décrit les étudiants chinois comme " silencieux ou même passifs » et Ghobain décrit (2016:49) les Saoudiens comme " passifs, dépendants et non autonomes » et souligne la dominance de l'approche centrée sur l'enseignant. Pareillement, Sonaiya (2002, cité dans Benson, 2006 : 25) dit

XLinguae, Volume 10, Issue 4, October 2017, ISSN 1337-8384, eISSN 2453-711X 
que l'autonomie n'est pas un concept utilisable en culture africaine. Selon certains auteurs, l'apprentissage centré sur l'enseignant est profondement ancré dans les systèmes éducatifs de l'Europe centrale et la centration sur l'apprenant s'impose avec beaucoup de difficulté (Hanesova, 2015 : 46). Selon nos observations, l'enseignement des langues dans le contexte slovène est fortement centré sur l'apprenant. Cela ne garantit bien sûr pas encore l'autonomie des apprenants, mais les enseignants y travaillent. Nous serions donc plutôt d'accord avec Little, qui a souligné que l'autonomie est une catégorie universelle en rejetant l'idée que l'autonomie est un objectif inapproprié dans des cultures non-occidentales (Cotterall, $2008: 2$ ).

La perception des rôles assumés par les apprenants et les enseignants subit, dès l'apparition de l'approche communicative, une profonde métamorphose, écrit Smuk (2014 : 43). Les apprenants et les enseignants sont tous deux actifs, comme le souligne Benson (2007 : 15), ils sont « engagés mutuellement, mais dans une perspective différente ». Depuis bien longtemps, l'enseignant n'est plus seulement le détenteur du savoir ; ses rôles se multiplient ; Cicurel (2013 : 167) reprend la taxinomie de Louise Dabène qui a, déjà en 1984, dégagé trois attributions pour le professeur : une fonction de vecteur d'information, une fonction de meneur de jeu et une fonction d'évaluateur. La liste des fonctions pourrait sans doute être complétée ; avec les changements de doctrine et le développement technologique, l'enseignant acquiert d'autres fonctions et devoirs auxquels il était impossible de penser il y a 30 ans, entre autres ceux de motivateur et celui qu'on pourrait appeler le rôle de conscientisateur.

Dans le processus du développement de l'autonomie de l'apprenant, l'enseignant peut avoir plusieurs rôles; selon Voller, (1997, cité dans Udier et al., 2016 : 104), il peut servir d'aide, de conseiller ou de ressource. L'enseignant - " aide » est tolérant, empathique, ouvert, ne juge pas et essaie de motiver les apprenants. Il tend à conscientiser ses élèves, les aide à planifier, à décider de leurs objectifs et à réfléchir sur le processus d'apprentissage. L'enseignant - conseiller réagit de manière semblable en ce qui concerne l'encouragement et la motivation, mais donne plus d'accent sur un apprentissage autonome. Et dernièrement, l'enseignant-ressource sert de point de repère quand les apprenants apprennent en autonomie (ibid.).

Dans le processus d'enseignement / apprentissage, les rôles de l'apprenant et de l'enseignant sont différents, mais l'apprenant n'est plus un consommateur passif des contenus, proposés par l'enseignant. Même si, selon un accord commun, c'est celui qui détient le savoir, donc l'enseignant, qui est responsable du processus pédagogique, ce processus devrait être le résultat d'une négotiation entre les deux partenaires (Siquiera Nicolaides, 2007 : 158). Ben-Zvi et Sfard (2007 : 117) parlent de la distribution du travail entre « ceux qui enseignent et ceux qui apprennent » et ajoutent que chaque interaction apporte une opportunité pour apprendre et pour enseigner (ibid.).

L'apprenant devient-il plus conscient de son apprentissage et donc plus autonome ? Little (1996 : 11-12) dit qu'en tant qu'apprenants d'une langue étrangère, sauf si nous l'apprenons dans la petite enfance, nous sommes toujours conscients de l'enseignement et de l'effort investi lors de l'apprentissage. Selon lui, la conscience de la necessité d'apprendre et l'effort utilisé sont indissociables de notre utilisation de la langue cible. Pour lui, un apprenant de langue étrangère est beaucoup plus conscient de son apprentissage qu'un locuteur natif (ibid : 20). Gautherot (2013:231) dit que, dès l'apparition des approches sur objectifs spécifiques et des approches communicatives, l'apprenant est considéré « non plus comme un utilisateur 'à venir' de la langue, mais dès l'entrée en classe, comme un utilisateur à part entière, fût-il débutant, et un usager en relation avec d'autres usagers, engagé dans un 'projet' langagier - en un mot, comme un 'acteur' ».

L'enseignant qui veut développer l'autonomie chez ses apprenants devrait aussi être capable de s'observer soi-même en tant qu'apprenant d'une langue étrangère et de réfléchir sur ses propres capacités en tant qu'apprenant autonome (Udier et al. 2016 : 
106). Dans son article, Benson (2007 : 16) constate que l'idée de l'autonomie est souvent représentée comme une idée centrée sur l'apprenant. Il décrit son expérience en tant qu'apprenant de la langue cantonaise qu'il a entreprise dans le but de mieux comprendre l'autonomie depuis la perspective de l'apprenant. Il part de l'hypothèse que les apprenants en général tentent de mener leur vie de façon autonome et s'intéressent à la contribution que leur apprentissage apporte à leur autonomie. Selon lui, la question que se posent les apprenants est la suivante : que puis-je faire pour atteindre le but de l'autonomie personnelle ? (2007 : 26). Après s'être observé, il conclut que le processus et les résultats supportent son autonomie personnelle. Il s'est trouvé autonome en ce qui concerne son apprentissage en général ; la plupart de ce qu'il a fait en tant qu'apprenant a été le résultat de ses choix et décisions. Cependant, il a manqué d'autonomie en étant trop dépendant de son enseignante quant au choix du contenu à apprendre : " J'apprends ce que mon enseignante pense que je devrais apprendre » (ibid. : 28).

J'ai tenté ${ }^{1}$, cette année, de faire quelque chose de semblable en m'inscrivant dans un cours de coréen pour débutants. J'ai choisi cette langue dans la perspective de prochains voyages en Corée du Sud, mais aussi en raison de ma fascination pour la culture coréenne. Ma situation n'a pas été tout à fait comparable à celle de Benson - je vis dans un milieu où la langue apprise est peu présente - cependant, il y avait quelques points communs, notamment le fait que, comme Benson, je ne suis probablement pas une apprenante typique et j'avais, moi aussi, très peu de temps à consacrer à cet apprentissage. J'ai constaté, après le cours, que j'étais très peu autonome. Contrairement à mes attentes et mes expériences avec les autres langues, je dépendais complètement de mon enseignante et suivais plus ou moins aveuglement son itinéraire d'apprentissage. Les quelques (rares) initiatives que j'ai entreprises pour apprendre en indépendance (en m'achetant un manuel complémentaire et en m'abonnant à quelques sites sur Internet) n'ont pas donné les résultats escomptés. Pourtant, cette expérience a été précieuse car elle m'a permis de mieux comprendre mes propres apprenants et la réfléxion et l'auto-observation pendant l'apprentissage m'ont permis de me mettre à leur place.

\section{La conscientisation}

Le concept de conscientisation n'est pas nouveau dans le monde de la pédagogie ; il est pourtant peu recherché dans le contexte slovène alors qu'il l'est davantage dans le contexte croate avoisinant, voir p. ex. les travaux de Vrhovac et ses collègues (2012). Freire (1969, cité dans Ferrer et Allard, 2002 : 99) définit la conscientisation comme « un processus social qui mène à l'approfondissement de la prise de conscience, à une conscience reflétant une connaissance plus vaste, à une conscience critique fondée sur le décodage des valeurs implicites et explicites contenues dans les informations recueillies ». Ferrer et Allard s'inspirent de sa définition et définissent la conscientisation critique qui est « le processus par lequel une personne détermine, observe et analyse les facteurs qui influent favorablement ou non sur sa vie, sur sa communauté, sur d'autres personnes et d'autres collectivités ainsi que sur l'environnement » (ibid. : 100). Dans ces deux artices, Plathner, quant à lui, discute de l'expression française conscientisation et la compare aux expressions, utilisées dans l'espace anglophone, consciousness-raising (Ellis, 1994, cité dans Plathner, 2009

${ }^{1} \mathrm{Vu}$ le fait qu'il s'agit d'une expérience (très) personnelle, je me permets d'écrire cette partie à la première personne du singulier.

XLinguae, Volume 10, Issue 4, October 2017, ISSN 1337-8384, eISSN 2453-711X 
: 46), conscious awareness (Schmidt, 1995, ibid.) et language awareness (Hawkins, 1999, ibid.). Il lui semble que le terme de conscientisation englobe les aspects contenus dans les termes anglais et apporte un renforcement des dimensions procédurale et sociale (ibid.). Il cite aussi les «partisans francophones des processus conscients » des années 70/80 du siècle passé ; Roulet et Boyer (Plathner, 2008 : 30, 2009 : 46) qui se sont, tous deux, intéressés à la réflexion métalinguistique, Boyer surtout en relation avec l'identification linguistique et la conceptualisation grammaticale.

Plathner attire aussi l'attention sur le fait que le terme " conscience » peut donner lieu à confusion parce qu'on peut distinguer plusieurs aspects de conscience : prise de conscience, conscience comme intention et conscience comme connaissance (Plathner, 2008 : 31). Le terme " conscience " désigne un etat et l'expression « conscientisation » désigne un processus, un travail sur la conscience de l'apprenant (ibid.).

Dans la plupart des cas, le mot conscientisation est utilisé en relation avec un thème, traité en classe, ou une structure grammaticale à acquérir ; Plathner, par exemple, parle de l'exemple du subjonctif en français. Dans notre étude, nous allons nous focaliser sur le processus d'apprentissage lui-même et non sur une question grammaticale concrète.

La conscientisation engage aussi bien l'apprenant que le guide (Plathner, 2008: 32), donc l'enseignant et l'apprenant. L'enseignant doit « amener les apprenants à réfléchir sur leurs besoins en termes de communication, les entraînant ainsi à une prise de conscience de leur apprentissage et à l'autonomie " (Cadre européen de référence pour les langues ${ }^{2}, 2001: 46$ ).

Le concept devient plus actuel avec la parution du CECRL en 2001. L'enseignement/apprentissage est encore plus authentique et l'apprentissage basé sur les tâches présuppose les apprenants plus autonomes et conscients de leur rôle dans le processus et capables de gérer, le plus possible, leur propre apprentissage. Ils doivent accepter la responsabilité de leur apprentissage et être capables d'y réfléchir (CECRL : 114).

\section{L'exemple des comptes-rendus}

\subsection{La démarche et l'hypothèse}

Depuis plusieurs années, nous proposons à tous nos groupes d'écrire des comptesrendus de nos cours. Nous y voyons plusieurs avantages, le premier étant le fait que tous les étudiants, y compris les absents, soient au courant de ce qui se passe en cours et des devoirs.

La rédaction de ces comptes-rendus est obligatoire et incluse dans la liste des obligations. Le devoir est effectué à tour de rôle ; après le premier cours, nous dressons la liste des noms des étudiants par ordre alphabétique que nous envoyons à tous les étudiants inscrits dans chacun de nos groupes. Nous proposons aussi la structure de base du compte-rendu à suivre ; simplifiée pour qu'elle puisse être suivie par les étudiants de première année qui n'ont pas tous un très bon niveau de langue. La structure de base est la suivante : date du cours et le numérotage, la description du contenu du cours (« Ce que nous avons fait»), les devoirs (« Ce qui est à faire jusqu'au prochain cours ») et l'évaluation du cours, partie facultative (« Mon avis »). La dernière partie est facultative car nous ne voulons pas trop les forcer à exprimer leur avis ; comme il s'est avéré lors de l'analyse, nous avions raison de ne pas instister, nous y reviendrons plus loin. Le devoir consiste donc à rédiger ce compte-rendu et à

${ }^{2}$ Dans la suite : CECRL. 
l'envoyer à tous les étudiants instrits et à l'enseignante, le lendemain du cours au plus tard. Si l'étudiant à qui c'était le tour, est absent, le suivant de la liste prend sa place c'est de la responsabilité des étudiants.

À partir de l'année dernière, nous avons introduit un changement car certains comptesrendus étaient truffés d'erreurs. L'étudiant a donc d'abord envoyé le compte-rendu à l'enseignante et a ensuite envoyé le compte-rendu corrigé à tout le monde.

Les comptes-rendus étaient assez différents ; certains très basiques, ne répondant que de façon très limitée aux contraintes et ne contenant que des alinéas, d'autres beaucoup plus développés et décrivant le cours dans le moindre détail. La longueur allait de 10 lignes à 2 pages et la différence entre les étudiants de lère année de licence et les étudiants en Master 1 était clairement visible.

À la fin du cours, nous avons demandé à tous les étudiants d'écrire une réflexion dans laquelle ils devaient évaluer l'activité exercée durant tout le cours. La consigne était d'inclure dans cette réflexion l'évaluation de l'activité. La longueur de la réflexion n'avait pas été spécifiée et ils étaient libres de l'écrire en français ou en langue maternelle. La consigne a volontairement été laissée assez ouverte ; nous voulions que les étudiants s'expriment librement et sans contraintes.

À la fin du cours, nous avons donc obtenu 50 réflexions de longueur inégale (allant d'une demi-page jusqu'à une page et demie). Elles ont été rendues de façon anonyme (un des étudiants de chaque cours s'est chargé de les recueillir, les anonymiser et nous les envoyer par la suite). 18 ont été écrites en français et 32 en langue maternelle.

Les étudiants ont suivi les cours suivants :

- $\quad 10$ le cours de Français en usage (1ère année de licence, année universitaire 2016/17)

- 13 le cours de Typologie textuelle (2ème année de licence, année universitaire 2015/16)

- 10 le cours de Didactique de FLE 1 (1ère année de Master, année universitaire 2015/16)

- 17 le cours de Didactique de FLE 1 (1ère année de Master, année universitaire 2016/17) ;

En tout donc 23 réflexions des étudiants en licence et 27 des étudiants en Master 1, ce qui nous a donné un corpus assez équilibré, concernant les deux niveaux d'études. Entre les deux groupes d'étudiants, il y a encore une différence significative : les étudiants en Master sont inscrits en filière pédagogique et se préparent donc à devenir enseignants.

Ce qui nous a intéressé dans ces réflexions, c'était de voir si les étudiants, en observant les cours, seraient capables de dégager la structure et de déterminer les objectifs des cours. Nous voulions aussi voir si dans la partie " votre avis » ils seraient capables de donner des suggestions et participer de cette manière activement à la conception des cours.

$\mathrm{Vu}$ que les étudiants en Master 1 ont terminé leurs études du 1er cycle, qu'ils ont choisi la filière pédagogique et se préparent donc à devenir enseignants de langue(s) ou de français et d'une autre matière ${ }^{3}$, nous considérions qu'ils seraient plus autonomes, dans le sens des deux premières caractéristiques, mentionnées par Dickinson (1993 : 330): l'apprenant autonome est conscient de ce que fait l'enseignant et est capable de reconnaître les objectifs de l'enseignement. Nous supposions aussi

${ }^{3}$ Les étudiants en filière pédagogique suivent un double cursus - le français et une autre matière (une autre langue ou une autre matière qui peut être étudiée à la faculté).

XLinguae, Volume 10, Issue 4, October 2017, ISSN 1337-8384, eISSN 2453-711X 
qu'il seraient plus conscients de leur rôle dans l'environnement dans lequel ils apprennent (Siquiera Nicolaides, 2007 : 141).

L' hypothèse de départ était donc la suivante : il y aurait dans les réflexions des étudiants de Master 1 plus de commentaires concernant le processus d'enseignement/apprentissage, ce qui montrerait qu'ils sont plus conscients de ce processus.

Pour analyser les réflexions, nous avons utilisé une démarche qualitative ; nous avons codé les réflexions en utilisant les abréviations suivantes : LIC1 et LIC2 respectivement pour les étudiants de 1ère et de 2ème année de licence et M1 pour les étudiants de première année de master.

\subsection{Les résultats de l'analyse}

Après la première lecture des réflexions, nous nous sommes rendu compte que quelques thèmes apparaissaient assez fréquemment dans les textes, dont quelques uns auxquels nous n'avions pas pensé. Nous avons codé les réflexions pour nous faciliter l'analyse.

Les thèmes récurrents sont : l'utilité de cet exercice, l'activité permet d'être informé, la formation du groupe, la pratique de l'expression écrite en français, l'apprentissage et la mémorisation du lexique, c'est un exercice dans la technique de la rédaction des comptes-rendus. Pour reprendre de manière plus schématique :

- l'utilité de l'activité - elle est/n'est pas utile,

- l'activité permet d'être informé, par exemple en cas d'absence,

- la formation du groupe (grâce à cette activité, on se connaît mieux, le groupe devient plus uni),

- $\quad$ apprentissage de nouveaux mots,

- pratique de l'expression écrite en français,

- amélioration de la technique du compte-rendu,

- conscientisation : on est obligé de réfléchir au contenu / aux objectifs des cours.

Dans la suite, nous reprendrons les opinions des étudiants. Parfois, elles se répètent pratiquement mot-à-mot, dans ce cas-là nous ne les citons qu'une seule fois.

\subsubsection{L'utilité de l'activité}

Tous les étudiants, sauf $u^{4}$, ont trouvé l'activité utile. Ils soulignent plusieurs avantages dont nous allons parler plus en détail dans la suite. L'étudiant qui n'a pas trouvé l'activité utile, dit : « les comptes-rendus sont surtout utiles pour les absents. Je ne vois pas pourquoi je devrais le faire pour les autres. Il y a déjà beaucoup d'autres devoirs à faire » $(\mathrm{M} .10)$

La grande majorité des étudiants soulignent le fait que cette activité permet d'être informé, surtout quand on est absent ; cet avis a été exprimé par 49 étudiants. Certains étudiants ont souligné que les comptes-rendus leur ont permis de toujours savoir ce qui était à faire et qu'ils les relisaient avant l'examen, pour se rappeler le contenu des cours.

\footnotetext{
${ }^{4}$ Les comptes-rendus étant anonymes, nous utilisons la forme masculine en tant que forme neutre, sauf parfois dans les citations des réflexions, quand il est possible de déduire le sexe de l'auteur.
} 
- Je savais toujours ce qui était pour le devoir. (LIC1.1)

- $\quad$ Si on n'est pas présent, on pourra se renseigner sur ce qui s'est passé en classe ce jour -là. Il en va de même pour le devoir. (LIC1.2)

- Les comptes-rendus sont utiles pour les absents ; de cette manière ils n'ont pas d'excuses et savent très bien ce qui est à faire. (LIC1.5)

- C'est la meilleure manière de rester renseigné pendant tout le semestre. (...) Les commentaires aident beaucoup. De cette manière il m'est plus facile de reconstituer le cours. (LIC2.2)

- Cela m'a aidé quand j'oubliais ce que je devais faire. (LIC2.4)

- C'est commode pour les personnes absentes. (LIC2.10)

- C'était utile pour les devoirs ; je note toujours ce qui est à faire, mais j'ai lu les comptes-rendus pour vérifier. (M1.1)

- Il y avait aussi toutes les informations concernant les observations de classes, le stage pédagogique etc. (M1.14)

- $\quad$ Si on était absent, on n'avait plus besoin d'appeler les collègues ; il fallait tout simplement attendre le compte-rendu pour être au courant de tout. (M1.18)

- $\quad$ Cela m'a aidé quand je n'étais pas assez attentif pendant le cours. (M1.19)

- J'ai beaucoup aimé cette activité à cause de ma nature distraite ; elle m'a permis de retrouver les informations importantes. (M1.23)

- Cela évite les drames et les publications nocturnes sur Facebook, par exemple : « je vous supplie, dites-moi ce qui est à faire pour demain et dans quelle salle on se retrouve ». (M1.26)

- $\quad$ Il n'y avait plus d'excuses du type : «J'étais absent », « Je ne savais pas que c'était à faire », « J'ai oublié ». (M1.21)

Un étudiant a aussi souligé l'importance des comptes-rendus pour l'enseignant :

- C'est aussi positif pour le professeur, qui, en lisant les comptes-rendus, peut se rappeler ce qu'on a fait pendant tout le semestre. (LIC2.7)

Les étudiants en maîtrise mentionnent souvent que les comptes-rendus les ont aidés à mieux se préparer pour l'examen.

- $\quad$ À la fin du semestre, nous avons eu tous les comptes-rendus sur nos mails, ce qui m'a permis de mieux m'organiser pour l'examen. (M1.7)

- $\quad$ On peut lire ces comptes-rendus en se préparant pour l'examen et de cette manière révise de façon systématique ce qu'on a fait au cours du semestre. (M1.17)

- $\quad$ Ils facilitent la préparation à l'examen. (M1.26)

\subsubsection{La formation du groupe}

Un étudiant en licence dit que l'activité lui a permis de mieux connaître ses collègues. Parmi les étudiants en maîtrise, cet avis est plus présent et exprimé par 6 étudiants.

- Les comptes-rendus ont permis aussi une meilleure compréhension et une bonne communication. (LIC2.3)

- Une fois enseignante, je vais aussi utiliser ce type de travail collaboratif. (M1.1)

- C'est surtout utile en première année de licence quand on ne connaît pas encore ses camarades. (M1.2) 
- Grâce à cette activité, on a bien travaillé ensemble, on avait l'impression de vraiment appartenir au groupe. (M1.23)

- Les comptes-rendus ont servi aussi à établir des liens entre nous, surtout au début quand on ne se connaissait pas encore très bien. L'activité nous a permis de connaître les prénoms et les noms de nos collègues et d'avoir leurs courriels. (M1.25)

- Il s'agit d'un travail de groupe car on pense tout le temps à tout le monde, aussi aux absents. (M1.26)

- Cela aide à souder le groupe, cela crée un sentiment d'appartenance. (M1.27)

\subsubsection{Une meilleure maîtrise du vocabulaire}

L'acquisition du vocabulaire a été uniquement mentionnée au niveau licence (7 étudiants) et uniquement par les étudiants de 1ère année.

- $\quad$ C'est utile pour répéter les mots clés, appris en cours. (LIC1.7)

- Je retiens mieux les mots appris en cours si je les vois encore une fois dans le compte-rendu. En plus, le vocabulaire se répète, par exemple les verbes comme lire, écrire, parler, présenter, envoyer, discuter, vérifier et d'autres mots comme le compte-rendu, la page, la feuille, le cours, premièrement, puis, à la fin, au début, etc. (LIC1.10)

\subsubsection{Exercice d'expression écrite, technique de rédaction des comptes- rendus}

4 étudiants au niveau licence ont mentionné que l'activité leur paraissait utile pour pratiquer l'expression écrite en français. Les étudiants en maîtrise l'ont mentionné plus souvent (7 étudiants).

Assez souvent (5 étudiants au niveau licence et 7 en Master) les étudiants mentionnent aussi que l'activité leur permet d'améliorer leur technique de rédaction des comptesrendus.

De cette façon, on est en quelque sorte obligés d'écrire en français. D'abord je n'étais pas habituée à écrire en français et j'ai trouvé la tâche difficile, mais maintenant je l'apprécie davantage. (LIC1.1)

- $\quad$ Un texte (court) à écrire, super exercice. (LIC1.7)

- $\quad$ Ecrire, c'est aussi bien pour améliorer notre expression écrite. (LIC2.3)

- J'ai eu l'opportunité d'améliorer ma façon d'écrire. (LIC2.7)

- De cette manière, on produit un texte en français. (M1.4)

- On pratique l'expression écrite et on commence à présenter le contenu des cours de façon structurée. (M1.10)

- Un bon exercice pour apprendre à présenter de façon concise les éléments les plus importants du cours. (M1.20)

- J'ai appris à écrire les comptes-rendus. (LIC1.1)

- De plus, en écrivant le compte-rendu, on s'entraîne à écrire un texte différent. (LIC2.7)

- Ecrire le compte-rendu était parfois difficile mais seulement parce que je n'étais pas habituée à le faire. (LIC2.11)

- C'est un exercice de synthèse ce qui est utile car les Français aiment beaucoup le faire. (M1.11)

- C'est un exercice de synthèse. (M1.15) 


\subsubsection{Conscientisation}

Dans cette partie, nous allons inclure tous les commentaires concernant le contenu du cours ; donc tous ceux qui montrent que les auteurs participent activement en classe et en sont conscients. Ces commentaires sont formulés de façon très variée et sont plutôt rares au niveau licence (6 étudiants). Les étudiants en Master s'expriment plus souvent sur le contenu des cours (14 étudiants, donc, plus de la moitié). Leurs remarques sont aussi plus élaborées.

- $\quad$ Cette activité nous force à suivre le cours. (LIC1.5)

- Quand c'est notre tour, on est obligé de suivre le cours et de participer tout le temps. (LIC2.2)

- Quand on écrit le résumé, on réfléchit sur la thématique, le contenu du travail, on répète le déroulement du cours. De cette façon on mémorise et on vérifie si on a compris le sujet du cours. (LIC2.6)

- La personne qui écrit le compte-rendu doit réfléchir aux tâches qu'on a faites. En réfléchissant, on se prépare déjà à l'examen. (LIC2.7)

- $\quad$ On est obligé de suivre le cours. (LIC2.12)

- C'est un bon exercice pour apprendre à être responsable et ponctuel (envoyer le compte-rendu à temps). LIC2.13

- C'est utile pour celui qui écrit le compte-rendu ; il doit faire attention à ce qu'on fait pendant le cours. (M1.3)

- Celui qui écrit résume brièvement le contenu du cours. (M1.4)

- On est attentif au déroulement et au contenu du cours ; ce qui peut nous aider à faire nos propres cours dans le futur. (M1.7)

- En écrivant, on reconstitue le cours et on le raconte ensuite de façon articulée. Parfois on se pose des questions à propos du contenu ou on a des idées pour amélioirer les choses. (M1.10)

- Quand c'est notre tour, on réfléchit tout le temps au contenu et au déroulement du cours, on se rend compte automatiquement de la structure du cours. À la fin, on doit être capable de faire une synthèse. Ces comptesrendus peuvent nous être utiles aussi comme orientation, pour mieux planifier nos cours dans le futur. (M1.12)

- Celui qui écrit doit suivre très attentivement et réfléchir au contenu du cours. (M1.21)

- $\quad$ On doit être attentif aux parties du cours et à la façon de travailler. (M1.22)

- $\quad$ À chaque fois, un étudiant est forcé de suivre le contenu et le déroulement du cours de façon structurée. (M1.24)

- On apprend à être responsable ; on écrit le compte-rendu pour soi-même, mais aussi pour les camarades. (...) Cette activité est, en fait, une analyse du cours. On ne s'en rend peut-être pas compte, mais en suivant le cours pour pouvoir écrire le compte-rendu, on observe le choix des activités et l'ordre dans lequel elles sont présentées. C'est une sorte de préparation pour notre futur travail. La partie du compte-rendu où on peut exprimer notre avis est un entraînement pour les évaluations de nos propres cours. (M1.25)

- Ce qui est bien, c'est qu'on doit vraiment suivre et parfois même se renseigner auprès de la professeure, pour voir si on a bien compris. On a donc une grande responsabilité car on ne voudrait pas transmettre de fausses informations à ses camarades. Pour pouvoir le faire, il faut aussi bien relire ses notes avant de l'écrire ; en faisant cela, on répète le contenu du cours. (M1.27) 


\subsubsection{Expression de l'avis concernant les cours}

Même si nous nous sommes attendu à plusiers commentaires à ce sujet, nous en avons trouvé seulement deux - les deux plutôt négatifs.

- En ce qui concerne les avis concernant les cours ; je les trouve assez inutiles. Je ne les ai jamais lus. On sait qu'on a du mal à exprimer une opinion critique. (LIC2.5)

- Je trouve bien qu'à la fin de chaque compte-rendu on a la possibilité d'exprimer notre avis concernant le cours. Mais ces avis sont toujours positifs; on sait qu'il est difficile d'émettre une critique. (M1.27)

\subsubsection{Les points à améliorer}

Dans leurs réflexions, les étudiants ont mentionné certains points qu'ils n'ont pas trop appréciés et qui sont, selon eux, à améliorer. Il s'agit surtout des consignes trop peu précises (les étudiants ne savaient pas toujours qui était chargé du compte-rendu et jusqu'à quand il fallait l'envoyer).

- $\quad$ On peut oublier de le faire à temps. (LIC1.1)

- Notre collaboration a parfois été assez pauvre ; on a fait preuve de négligence en ce qui concerne la remise du compte-rendu dans les délais fixés. (LIC2.10)

- Nous avons eu eu quelques problèmes en nous organisant car nous ne savions pas toujours à qui était le tour. Le mieux serait de le signaler au début de chaque cours pour que cet étudiant puisse prendre des notes plus détaillées. (M1.6)

- $\quad$ On devrait spécifier de façon plus claire quels sont les délais pour l'envoyer. (M1.8)

- Ce que je n'ai pas trop aimé, c'est que certains les ont écrits de façon vraiment trop détaillée. (M1.18)

\section{Discussion}

Après l'analyse, nous pouvons constater que la rédaction des comptes-rendus a été perçue comme utile par 49 étudiants, donc par tous, sauf un. Celui qui ne l'a pas apprécié l'a évaluée en tant qu'étant utile surtout pour les absents et n'y a vu qu'un travail supplémentaire, inutile. Les étudiants ont mentionné que les comptes-rendus leur ont permis d'être informés quand ils étaient absents, mais aussi quand ils n'ont pas suivi le cours très attentivement. Cette remarque a été plus souvent exprimée par les étudiants en Master ce qui est assez logique car ils ont plus d'obligations que les étudiants en licence (plus de devoirs - aussi facultatifs et plus d'autres obligations, par exemple les observations des classes et le stage pédagogique).

Sept étudiants ont mentionné que l'activité a servi à la formation du groupe, un en licence et 6 en master. Écrire et envoyer les comptes-rendus à ses camarades présuppose qu'on a les contacts (dans notre cas les courriels) de tout le groupe, ce qui devrait aider les étudiants à mieux communiquer entre eux, surtout quand ils ont besoin de quelque chose ou quand il y a un devoir ou une tâche à faire en groupe. Il se peut que les étudiants préfèrent d'autres moyens de communication - par exemple, des groupes sur des réseaux sociaux où ils se sentent moins contrôlés et donc plus à l'aise. Les étudiants en Master se rendent probablement plus compte de l'importance du groupe pour mieux travailler; certains mentionnent aussi le sentiment d'appartenance au groupe.

Sept étudiants en licence ont souligné que les comptes-rendus les ont aidés à apprendre de nouveaux mots ou à mieux mémoriser les mots qui se répétaient souvent. En préparant l'activité, nous n'y avons pas pensé et ce point n'a pas été 
mentionné par les étudiants en Master. Leurs remarques sont probablement dues au fait que les comptes-rendus des étudiants de premier cycle ont été rédigés de façon plus basique et la structure proposée a été plus respectée ; pour cela certains mots se répétaient souvent et les étudiants, surtout ceux ayant un niveau plus faible, en ont profité.

Ce qui nous a plus surpris, est le fait que les étudiants ont mentionné la pratique de l'expression écrite et que cela a été mentionné plus souvent par les étudiants en Master que par les étudiants en licence. Cela peut paraître surprenant au premier abord, mais ne l'est pas ; à cause des cours généraux de pédagogie et psychologie, les étudiants en filière pédagogique ont très peu de cours de langue et ils le ressentent comme un point très négatif.

Les étudiants mentionnent aussi la technique, 5 étudiants du premier cycle et 7 étudiants en Master ont écrit que cette activité leur a permis de s'exercer à écrire les

comptes-rendus. À notre avis, il s'agit d'une question culturelle ; en langue maternelle, ce type d'écrit est plutôt rare et n'est pas souvent pratiqué dans le contexte scolaire, c'est pourquoi les étudiants le perçoivent comme quelque chose de difficile et de très 'français'.

Ce qui nous a le plus intéressé dans les réflexions, c'était de voir à quel point les étudiants perçoivent les comptes-rendus comme un moyen de conscientisation. Les commentaires concernant le contenu et les objectifs des cours sont plus souvent exprimés par les étudiants en Master (6 étudiants au niveau licence et 14 étudiants au niveau Master). Certains étudiants disent que l'activité les a forcés à suivre le cours et à être plus attentifs (cette opinion a été exprimée le plus souvent). Certains étudiants en Master voient l'activité aussi comme une préparation pour leur futur travail d'enseignants et commentent qu'en suivant le cours, ils ont mieux observé l'organisation des cours, la fixation des objectifs et la planification du temps. Trois étudiants disent aussi qu'à l'aide de cette activité ils ont appris à être plus responsables et un de ces trois mentionne aussi qu'il fallait être ponctuel.

Les étudiants se sont, par contre, rarement exprimés sur la partie du compte-rendu où ils avaient la possibilité d'exprimer leur avis et de donner des suggestions concernant le cours. Cette partie n'a pas été mentionnée par les étudiants en licence et a été mentionnée deux fois seulement par les étudiants en Master - les deux fois de façon assez négative. Les étudiants écrivent qu'ils ont du mal à exprimer une critique, envers l'enseignant ou envers leurs collègues.

Notre hypothèse de départ est donc confirmée car les étudiants en Master se sont exprimés plus souvent sur le contenu et les objectifs des cours et sont donc plus conscients du processus d'enseignement/apprentissage.

\section{Propositions d'amélioration}

Après cette analyse, les améliorations s'imposent. L'activité a été perçue comme utile par pratiquement tous les étudiants mais dans les réflexions on voit clairement que les étudiants de licence et ceux de Master la perçoivent différemment.

Avec les étudiants en licence, il vaut mieux développer le côté linguistique et le point « information » qu'ils avaient mentionné comme avantages. Il y a aussi certains points techniques à améliorer : élaborer les consignes (déterminer de façon plus exacte la structure, l'ordre dans lequel les étudiants écrivent les comptes-rendus et surtout le délai dans lequel le compte-rendu doit être envoyé aux camarades du cours). Des textes plus longs seraient aussi un avantage car ils permettraient aux étudiants d'écrire plus en français.

Il y aurait plus de changements à introduire en cours de Master, pour les futurs enseignants de français - pas nécessairement tous liés à la rédaction des comptesrendus. Comme le dit Ok (2016 : 67), il est extrêmement important de développer l'autonomie des futurs enseignants pour les aider ainsi dans leur travail futur. Pour

XLinguae, Volume 10, Issue 4, October 2017, ISSN 1337-8384, eISSN 2453-711X 
pouvoir former des « practiciens réflexifs » (voir p. ex. Estrela, 2001), il faut que nos étudiants apprennent à valoriser la théorie à laquelle ils sont exposés en formation initiale (et qu'ils critiquent souvent, en disant qu'elle est inutile) et apprennent à en réfléchir et à l'utiliser en pratique.

Le premier changement que nous allons introduire à partir de cette année universitaire n'est pas directement lié à la rédaction des comptes-rendus mais il va très probablement aider les étudiants à réfléchir sur leur autonomie. Depuis plusieurs années déjà, nous organisons, avec les collègues du Département d'allemand de notre faculté ce que nous appelons "un enseignement réciproque »; les étudiants d'allemand enseignent l'allemand aux étudiants de français et vice versa. L'expérience n'est pas tout à fait semblable à celle de Benson ou à notre expérience - nous avons décrit les deux au début de l'article - car l'allemand est plus présent et sans doute plus facile à apprendre pour les locuteurs slovènes que le cantonais ou le coréen. Pourtant, nous allons profiter de cette expérience pour inciter la réflexion à propos de leur autonomie en classe de langue, en tant qu'apprenants. Nous tentons de le faire à l'aide de journaux de bord avec des consignes pour l'auto-observation, données à l'avance.

Le deuxième changement à faire consisterait à transformer la rédaction des comptesrendus en activité

d'apprentissage collaboratif, ce qui apporterait une plus-value à cette activité. Comme le soulignent Melnichuk et Osipova (2017 : 27), en pratiquant l'apprentissage collaboratif, les apprenants ont la possibilité de discuter avec les camarades, de présenter et défendre leurs idées, échanger et questionner les cadres conceptuels. Nous avons déjà utilisé la classe virtuelle sur la plate-forme Moodle pour le cours de Didactique du FLE 1, mais surtout pour y publier les documents, utilisés en cours. Nous envisageons d'y transférer aussi les comptes-rendus. L'utilisation de cette plateforme pour la publication des comptes-rendus permettra non seulement d'avoir tous les textes réunis à la fin de l'année mais donnera aux étudiants aussi la possibilité de commenter et d'échanger à propos du contenu des comptes-rendus et des devoirs (ou autres obligations) à faire. Avec un effort supplémentaire de la part de tous les participants, la classe virtuelle permettra aussi un apprentissage en autonomie, à l'exemple des cours de langue tatare en ligne (voir Gimaltedinova et Khalitova, 2016) et servira aussi bien, espérons-le, d'outil de conscientisation que d'outil d'encadrement de l'apprentissage (voir Temperman et al., 2007).

Cela nous mène à notre dernière réflexion. Plusieurs étudiants de Master se sont exprimés sur la partie « avis » des comptes-rendus en disant qu'il leur était difficile de s'exprimer. Il faudrait, à notre avis, discuter avec eux de la manière dont on peut exprimer une opinion, surtout critique, sans vexer le destinataire. Cela présuppose, bien sûr, que les étudiants aient confiance en leurs camarades de classe et en leur enseignant. Peut-être faudra-t-il les munir aussi de moyens linguistiques leur permettant une argumentation plus efficace. En même temps, cette partie leur donnera l'occasion de participer de façon plus active et de donner leurs suggestions quant au contenu du cours.

\section{Conclusion}

Dans le présent article, nous discutons de l'autonomie des apprenants au niveau universitaire, surtout en suivant les définitions de Holec et de Little, en supposant donc qu'un apprenant autonome est celui qui est capable de se charger de son propre apprentissage et d'avoir une attitude critique envers le processus pédagogique et le contenu des cours. Pour conscientiser les apprenants, nous avons utilisé depuis plusieurs années maintenant, un outil simple, les comptes-rendus des cours, écrits par les étudiants. Cette activité a été utilisée avec les étudiants au niveau licence et ceux au niveau Master, futurs enseignants en formation initiale. 
Les comptes-rendus se sont avérés être une activité utile. Dans leurs réflexions, les étudiants ont mentionné plusieurs avantages. Ils ont surtout aimé le fait d'être informés en cas d'absence, mais aussi la possibilité d'améliorer leur compétence linguistique (le vocabulaire, l'expression écrite, la technique du compte-rendu), certains ont aussi mentionné la formation du groupe. Les opinions des étudiants de licence et de Master différaient, ce qui a validé notre hypothèse de départ ; les étudiants en Master étaient, comme prévu, plus conscients du processus pédagogique et du contenu des cours.

Pour développer l'autonomie des étudiants et les conscientiser, nous proposons de travailler différemment avec les deux groupes. Avec les étudiants en licence, on devrait d'abord travailler la compétence linguistique ; un meilleur niveau de langue leur permettrait d'être plus autonomes en poursuivant leurs études. Avec les futurs professeurs en formation initiale, nous entamerons d'abord un travail d'autoobservation, en suivant les cours d'une langue étrangère encore inconnue. Ce travail leur permettra ensuite une réflexion sur les stratégies du développement de l'autonomie. Quant aux comptes-rendus, nous allons les transférer dans la classe virtuelle et essayer d'en faire une activité collaborative - pour permettre aux étudiants de participer activement lors des cours.

\section{Bibliographic references}

ADAMSON, J. - SERT, N. 2012. Autonomy in learning English as a foreign Language. In : International Journal of Global Education, vol. 1/2, pp. 23-27. ISSN 2146-9296.

BENSON, Ph. 2006. Autonomy in language teaching and learning. In : Language Teaching n. 40, pp. 21-40. ISSN 1475-3049.

BENSON, Ph. 2007. Teachers' and learners' perspectives on autonomy. In : LAMB. T. - REINDERS, H. (eds). : Learner and teacher autonomy, pp. 15-31. Amsterdam/Philadelphia: John Benjamins Publishing Company. ISBN 97890272 05179.

BEN-ZVI, D. - SFARD, A. 2007. Ariadne's thread, Daedalus' wings and the learners autonomy. In : Education et didactique, vol. 1, n. 3, pp. 117-134. ISSN 2111-4838.

CADRE EUROPEEN COMMUN DE REFERENCE POUR LES LANGUES. 2001. Paris : Conseil de l'Europe / Les Editions Didier. ISBN 227805075-3.

CICUREL, F. 2013. Culture professorale et singularité : une lecture de Bergson pour aborder la fabrique de l'action d'enseignement. In : BEACCO, J.-Cl. (éd.) : Ethique et politique en didactique des langues - Autour de la notion de responsabilité, pp. 165185. Paris : Didier. ISBN 9782278072651.

COMERIO, G. 2014. Supporting Language Learners Autonomy in Higher Education: The Self-Study Hour at the University of Nottingham Ningbo China. In : The Asian Conference on Language Learning 2014, Official Conference Proceedings, pp. 453463. ISSN 2186-4691.

CUQ, J.P. - GRUCA, I. 2002. Cours de didactique du français langue étrangère et seconde. Grenoble : PUG. ISBN 2706110821.

COTTERALL, S. 2008. Autonomy and Good Language Learners. In : GRIFFITHS, C. (éd) : Lessons from Good Language Learners. Cambridge : CUP. https://www.researchgate.net/publication/287113039_Autonomy_and_good_language _learners, consulté le 10 juin 2017

DICKINSON, L. 1993. Talking Shop, Aspects of autonomous learning. An interview with Leslie Dickinson. In : ELT Journal 47, n. 4, pp. 330-335. ISSN 0951-0893.

ESTRELA, M. T. 2001. Pratiques réflexives et conscientisation. In : Carrefours de l'éducation n. 12, pp. 56-65. ISSN 1262-3490.

FERRER, C. - ALLARD, R. 2002. La pédagogie de la conscientisation et de l'engagement : pour une éducation à la citoyenneté démocratique dans une perspective

XLinguae, Volume 10, Issue 4, October 2017, ISSN 1337-8384, eISSN 2453-711X 
planétaire. In : Education et francophonie, vol. XXX, n. 2, pp. 96-134. ISSN 19168659.

GAUTHEROT, J.M. 2013. Si la didactique des langues faisait vraiment sien « le politique ». In : BEACCO, J.Cl. (éd.) : Ethique et politique en didactique des langues - Autour de la notion de responsabilité, pp. 229-240. Paris : Didier. ISBN 9782278072651.

GHOBAIN, E.A. 2016. Envisaging an Approach to Promoting ESP Learners' Autonomy . In : Journal of Applied Linguistics (Dubai), n. 2(1), pp. 48-59. ISSN 2413-1105.

GIMALETDINOVA, G. - KHALITOVA, L. 2016. Self-paced learning: Investigating an online Tatar language course. In : XLinguae vol. 9, n. 3, pp. 81-92. ISSN 2453$711 \mathrm{X}$.

HANESOVA, D. 2015. A Move toward Learner-Centred Foreign Language Pedagogy: Focusing on Factors Fostering Language Intake. In : XLinguae vol. 8, n. 4, pp. 46-59. ISSN 2453-711X.

HOLEC, H. 1979. Autonomie et apprentissage des langues étrangères. Paris : Hatier. ISBN 2.218.06033.7.

LITTLE, D. 1991. Learner autonomy. 1: Definitions, issues and problems. Dublin : $\begin{array}{llllll}\text { Authentik. } & \text { ISBN } & 1 & 871730 & 02 & 3 .\end{array}$ https://www.researchgate.net/publication/259874253_Learner_Autonomy_1_Definiti ons_Issues_and_Problems, consulté le 10 juin 2017

LITTLE, D. 1996. Strategic competence considered in relation to strategic control of the language learning process. In : HOLEC, H. - LITTLE, D. - RICHTERICH, R. (éds) : Strategies in language learning and use, pp. 9-37. Strasbourg : Council of Europe Publishing. ISBN 92-871-2771-9.

MELNICHUK, M. V. - OSIPOVA, V. M. 2017. Cooperative Learning as a Valuable Approach to Teaching Translation. In : XLinguae vol. 10, n. 1, pp. 25-33. ISSN 2453$711 X$.

NUNAN, D. 1997. Designing and adapting materials to encourage learner autonomy. In : BENSON, P. - VOLLER, P. (éds.). Autonomy and independence in language learning. Londres : Longman, pp. 192-203. ISBN 0582289920.

OK, S. 2016. Autonomy in an EFL Teacher Training Context: Trainee Teacher Perceptions of Instructor Expectations. In : Australian Journal of Teacher Education, Volume 41, n. 3, pp. 65-86. ISSN 1835-517X.

PLATHNER, J.G. 2008. La conscientisation dans la classe de français avec l'exemple du subjonctif. In : Synergies Pays Scandinaves n. 3- 2008, pp. 27-44. ISSN 22612807.

PLATHNER, J.G. 2009. La «conscientisation» dans la classe de français : Compterendu d'une étude empirique. In : ForumSprache n. 1/2009, pp. 44-60. ISSN 18680852 .

SIQUEIRA NICOLAIDES, CH. 2007. Roles learners believe they have in the development of their language learning - autonomy included? In : LAMB. T. REINDERS, H. (eds). Learner and teacher autonomy, pp. 141-160. Amsterdam/Philadelphia: John Benjamins Publishing Company. ISBN 97890272 05179.

SMUK, M. 2104. Le savoir-être de l'apprenant au service du processus d'autonomisation. In : Synergies Chine, n. 9-2014, pp. 41-51. ISSN 2260-6483.

TEMPERMAN, G. - DE LIEVRE, B. - DEPOVER, CH. 2007. D'un outil d'awareness à un outil d'encadrement de l'apprentissage. In : Revue internationale des technologies en pédagogie universitaire vol. 4, n. 1, pp. 38-46. ISSN 1708-7570.

UDIER, S. L. - CILAS MIKULIC, M. - GULESIC MACHATA, M. 2016. Razvijanje ucenicke autonomije ucenja u hrvatskome kao stranom jeziku. In : CILAS MIKULIC, M. - BANKOVIC MANDIC, I. - JURCIC, A.T. (éds.) : Croaticum, 
Savjetovanje za lektore hrvatskoga kao inoga jezika, 3. zbornik radova. Zagreb: FF press, pp. 101-116. ISBN 978-953-175-597-9.

VIEIRA, F. - BARBOSA, I. - PAIVA, M. - FERNANDES, I. S. 2008. Teacher education towards teacher (and learner) autonomy. In: LAMB, T. - REINDERS, H. (eds.) : Learner and Teacher Autonomy, pp. 217-235. Amsterdam/Philadelphia: John Benjamins Publishing Company. ISBN 978902720517.

VRHOVAC, Y. (ed.) : 2012. Introduire le Portfolio europeen des langues dans les classes croates et françaises de langues etrangeres. De l'usage à la conscience linguistique. Zagreb : FF Press. ISBN ISBN 978-953-175-425-5.

WENDEN, A. 1991. Learner Strategies for Learner Autonomy. New York : Prentice Hall. ISBN 0-13-529603-X

Words: 8566

Characters: 54389 (30,22 standard page)

Ass. Prof. Dr. Meta Lah

Department of Romance languages

Faculty of Arts

University of Ljubljana

Aškerčeva 2, 1000 Ljubljana

Slovenia

meta.lah@ff.uni-lj.si 